\title{
Electron microscopic observations in perfusion-fixed human non-alcoholic fatty liver disease biopsies
}

\author{
Pauline Verhaegh ${ }^{1,2, *}$, Eddie Wisse ${ }^{3, *}$, Toon de Munck ${ }^{1,2}$, \\ Jan Willem Greve ${ }^{4,5}$, JoAnne VerheiJ ${ }^{6}$, Robert Riedl ${ }^{7}$, Hans Duimel ${ }^{3}$, \\ Ad Masclee ${ }^{1,2}$, Daisy Jonkers ${ }^{2}$, Ger Koek ${ }^{1,2,8}$ \\ ${ }^{1}$ Department of Internal Medicine, Division Gastroenterology-Hepatology, Maastricht \\ University Medical Center, Maastricht, the Netherlands; ${ }^{2}$ School of Nutrition and \\ Translational Research in Metabolism (NUTRIM), Maastricht University, Maastricht, the \\ Netherlands; ${ }^{3}$ Division of Nanoscopy, Maastricht Multimodal Molecular Imaging Institute, \\ and Department of Internal Medicine/Hepatology, The University of Maastricht, Maastricht, \\ the Netherlands; ${ }^{4}$ Department of Surgery, Zuyderland MC, Heerlen, the Netherlands; \\ ${ }^{5}$ Department of Gastro-Intestinal Surgery, Maastricht University Medical Center, Maastricht, \\ the Netherlands; ${ }^{6}$ Department of Pathology, Amsterdam University Medical Center, \\ Amsterdam, the Netherlands; ${ }^{7}$ Department of Pathology, Zuyderland MC, Heerlen, the \\ Netherlands; ${ }^{8}$ Department of Visceral and Transplantation Surgery, Klinikum RWTH, \\ Aachen, Germany; * these authors contributed equally to this work
}

\begin{abstract}
Summary
Non-alcoholic fatty liver disease (NAFLD) is a widespread liver disease in Western society, but its multifactorial pathogenesis is not yet fully understood. Ultrastructural analysis of liver sinusoidal endothelial cells (LSECs) in animal models and in vitro studies shows defenestration early in the course of NAFLD, promoting steatosis. LSECs and fenestrae are important in the transport of lipids across the sinusoids. However, human ultrastructural data, especially on LSECs and fenestrae, are scarce. This study aimed to explore the ultrastructural changes in perfusion type fixed liver biopsies of NAFLD patients with and without nonalcoholic steatohepatitis (NASH), with a special focus on LSECs and their fenestration. Liver biopsies from patients with NAFLD were fixed using two perfusion techniques, jet and injection fixation, for needle and wedge biopsies, respectively. Ultrastructural changes were studied using transmission electron microscopy. NASH was diagnosed by bright-field microscopy using the SAF score (steatosis, activity, fibrosis). Thirty-seven patients were included, of which 12 (32.4\%) had NASH. Significantly less defenestration was found in NASH compared to noNASH samples ( $p=0.002)$. Other features, i.e., giant mitochondria and fenestrae size did not differ between groups. Furthermore, we described new structures, i.e., single cell steatonecrosis and inflammatory fat follicles (IFF) that were observed in both groups. Concluding, defenestration was more common in noNASH compared to NASH in human liver samples. Defenestration was not related to the degree of steatosis or fibrosis. We speculate that defenestration can be a protective mechanism in simple steatosis which is lacking in NASH.
\end{abstract}

Key words: Non-alcoholic steatohepatitis; fenestrae; liver sinusoidal endothelial cell; defenestration.

Received 17 March, revised 23 July, accepted 29 July 2020 Available online 2 November 2020

\section{INTRODUCTION}

Non-alcoholic fatty liver disease (NAFLD) is the most widespread liver disease in Western society, with a prevalence up to $25 \%$. $^{1}$ It is strongly associated with overweight and obesity. ${ }^{2,3}$ The term NAFLD comprises both steatosis and non-alcoholic steatohepatitis (NASH). ${ }^{4} \mathrm{NASH}$ is an inflammatory state that develops in about $30 \%$ of NAFLD patients with significantly increased morbidity and mortality due to progression to fibrosis, cirrhosis and hepatocellular carcinoma (HCC). ${ }^{5,6}$

The pathophysiology of NASH is still poorly understood and is considered to have a multifactorial aetiology, involving dietary factors, host genetics, insulin resistance, lipotoxicity and microbiome perturbations. ${ }^{7}$ Studying ultrastructural changes in human liver biopsies may help to provide leads to further elucidate the pathophysiology of NAFLD.

Only a limited number of human studies have been performed that focused on ultrastructural changes in NAFLD. These studies showed autophagic vesicles ${ }^{8}$ and lipolysosomes in liver biopsies of NAFLD patients, ${ }^{9}$ with giant lipolysosomes in case of severe steatosis. ${ }^{9}$ Furthermore, Mallory-Denk body formation, 'cellular enlargement' and dilatation of the endoplasmic reticulum have been observed in NASH patients. ${ }^{10}$ Mitochondria have also been studied because of their role in beta-oxidation, showing crystalline inclusions and giant mitochondria in some but not all cases of NASH. ${ }^{11,12}$ Ahishali et al. reported larger mitochondrial diameter in NASH livers compared to fatty livers, but did not report a difference in the presence of giant mitochondria comparing NASH and fatty liver samples. ${ }^{13}$ However, a shortcoming of studies on ultrastructural changes is the rather small numbers of NAFLD and/or NASH patients included, ranging from four to 23, and only one study made a direct comparison of adult NAFLD patients with versus without NASH. ${ }^{13}$ Animal models and in vitro studies have also revealed interesting ultrastructural findings in NAFLD, especially with regard to liver sinusoidal endothelial 
cells (LSECs). LSECs are highly specialised endothelial cells at the interface between blood derived from the visceral adipose tissue and the gut on one side and hepatocytes and hepatic stellate cells on the other side. They have a unique phenotype, they lack a basement membrane and have fenestrae organised into sieves that regulate the transport of macromolecules, including lipids and lipoproteins across the sinusoid. ${ }^{14}$ Studies in animal models and in vitro studies have shown that the diameter and porosity of fenestrae show a negative correlation with excessive lipid ${ }^{15,16}$ and endotoxin ${ }^{17}$ exposure. A study in mice, deficient of plasmalemma vesicle associated protein (PLVAP), a protein coding gene required for the formation of fenestrae in LSECs, showed development of extensive multivesicular steatosis, steatohepatitis and fibrosis at around 3 weeks of life. ${ }^{18}$ This suggests that loss of fenestrae (defenestration) of LSECs may be an early step in the course of NAFLD. ${ }^{14}$ Due to the limited preservation of LSECs and their fenestrae by immersion fixation, as used in previous human electron microscopy studies, data on LSECs and their fenestrae in human samples is scarce. ${ }^{14}$ During immersion fixation, the fixative penetrates the tissue by diffusion, on its way fixating the tissues from the periphery to the centre. This leaves the centre of the tissue unfixed for almost an hour ${ }^{19}$ and only allows studying the well-fixed peripheral parenchymal cells. Sinusoidal cells and sinusoids collapse and lose details due to this improper fixation. ${ }^{20}$ To overcome this problem, we developed perfusion fixation methods for needle and wedge biopsies, ${ }^{19,20}$ which significantly improved the preservation of the sinusoids, sinusoidal cells and endothelial fenestrae, enabling high resolution transmission electron microscopy (TEM) of all hepatic cell types in human liver biopsies. Depending on the size of the tissue sample, wedge versus needle liver biopsy, different perfusion fixation techniques need to be used, namely injection $^{19}$ and jet ${ }^{20}$ fixation, respectively. Our aim was to explore the ultrastructural changes in human liver biopsies of NAFLD patients with and without NASH, with a special focus on LSECs and their fenestration. We hypothesised that fenestrae size and number decrease as the severity of NAFLD increases.

\section{METHODS}

\section{Patients and tissue sampling}

For the current explorative study, samples were available from patients with NAFLD undergoing a liver biopsy either for clinical reasons or as part of a research project. From all participants paraffin embedded needle biopsies were available for standard bright-field microscopy evaluation. Needle liver biopsies were obtained using a 16G needle and a biopsy gun (Bard Magnum Reusable Core Biopsy System; Bard, USA). Additionally, biopsy samples were collected for transmission electron microscopy (TEM) analysis. Liver wedge biopsy specimens were taken from 19 patients with NAFLD between October 2016 and October 2017 in the Zuyderland Medical Center, The Netherlands. Before inclusion, NAFLD had been diagnosed using imaging. The samples were collected during surgery [cholecystectomy $(n=1)$ and bariatric surgery $(n=18)$ ] as part of a study identifying non-invasive markers for NASH. This study was approved by the Medical Ethics Committee Zuyd and registered at clinicaltrials.gov (NCT02717000). All participants gave written informed consent prior to participation. Another 20 needle liver biopsies for TEM analysis were available as residual material from routine liver biopsies obtained from patients with a diagnosis of NAFLD based upon routine histopathological evaluation for clinical reasons at Mastricht University Medical Centre (MUMC+; The Netherlands) between 2011 and 2016. The Medical Ethics Committee AzM/UM had approved the use of the samples for the present study. The current study was performed according the declaration of Helsinki (latest amendment of 2013, Fortaleza, Brazil).

\section{Clinical evaluation}

The medical history, body mass index (BMI) and routine biochemical tests including bilirubin, alkaline phosphatase (AP), gamma-glutamyl transferase (GGT), alanine aminotransferase (ALAT), aspartate aminotransferase (ASAT), fasting glucose, ferritin, total cholesterol, triglycerides, high density lipoproteins (HDL) and low density lipoproteins (LDL), were collected prospectively for the patients from the Zuyderland Medical Center using standardised registration forms, and by retrospective review of the patients' medical files for the clinical MUMC+ patients.

\section{Bright-field microscopy}

Liver needle biopsies for bright-field microscopy analysis were fixed in $4 \%$ formalin and embedded in paraffin. Hematoxyline and eosin stained sections were used for NAFLD scoring according to the steatosis, activity and fibrosis (SAF) score ${ }^{21}$ by an experienced liver pathologist blinded to the clinical context and laboratory parameters. NASH was defined by score $A \geq 2$ including at least one point for ballooning and one point for inflammation. A score of $\mathrm{A}<2$ or the absence of ballooning was defined as 'noNASH'. 21

\section{Transmission electron microscopy (TEM)}

TEM analysis was performed on needle and wedge liver biopsies. From 19 patients, wedge biopsies were obtained during surgery at Zuyderland Medical Center. A portion of approximately $5 \times 5 \mathrm{~mm}$ was subjected to injection fixation for TEM analysis, according to the method described by Wisse et al. ${ }^{19}$ In short, a needle is used to inject the fixative into the wedge biopsy at a rate of approximately $1 \mathrm{~mL} / \mathrm{min}$. As injection of the fixative is not possible in needle biopsies, due to the smaller size, the technique of jet fixation as described by Vreuls et al. was used in 20 needle biopsy samples. This technique sprays the fixative with a constant pressure onto the liver tissue. ${ }^{20}$

All perfusion fixed biopsies were studied by TEM (FEI Tecnai G2 Spirit BioTWIN iCorr microscope; FEI Company, USA) at the Nanoscopy Unit of Maastricht University (M4I). The magnification was calibrated with a $463 \mathrm{~nm}$ line grid, before representative images of each biopsy were taken. Measurements of fenestrae diameter were done with Image $\mathbf{J}$ software (Rasband; National Institutes of Health, USA). Defenestration was defined by none or hardly any fenestrae found in well-fixed livers with preserved fine structured details, after scrutinising the whole samples.

\section{Statistical analysis}

Patient characteristics and ultrastructural findings were reported as absolute numbers and percentages for categorical variables and as mean with standard deviation (SD) or median and range, according to the normality of the distribution, for continuous variables. Findings were compared between NAFLD patients with and without NASH according to the SAF score using the independent t-test for normally distributed and the Mann-Whitney $U$ test for non-normally distributed data. Differences in dichotomous variables between groups were tested using the Chi-square test with Fisher exact when necessary. A two-sided $p$ value of $<0.05$ was considered statistically significant. All calculations were performed using SPSS version 24 statistical software (IBM Statistics for Macintosh; IBM, USA).

\section{RESULTS}

\section{Baseline characteristics}

In total, 19 wedge biopsy samples and 20 needle biopsy samples were available for TEM evaluation and 39 paraffin embedded needle biopsies for standard bright-field microscopic evaluation.

One needle biopsy sample was of insufficient quality to perform reliable bright-field microscopic scoring and was therefore excluded. All other biopsies contained a sufficient number of portal tracts to allow for correct evaluation of the hepatic architecture. With regard to the TEM samples, one needle biopsy was of insufficient quality for evaluation by TEM and therefore was excluded. 
Finally, biopsies of 37 patients were included in the analysis (Supplementary Fig. 1, Appendix A; flowchart of patient routing). Patient characteristics, BMI, routine biochemical test results at time of inclusion and bright-field microscopic results for the total group and subgroups (NASH/noNASH) are presented in Table 1.

All patients had NAFLD on initial histological evaluation or imaging, which was confirmed in 30 of them upon revision of the biopsy specimen for the current study. Twelve patients were classified as having NASH according to the SAF score. None of the patients had cirrhosis (F4) or overt pathology other than NAFLD on bright-field microscopic analysis. Patients with NASH showed higher AP, ALAT and ASAT levels as compared to noNASH patients (all $p<0.05$ ).

\section{TEM analysis of liver sinusoids}

An overview of the TEM findings comparing NASH with noNASH samples is given in Table 2 and findings of individual patients are presented in Supplementary Table 1 (Appendix A). The results of both jet and injection fixation are comparable and meet the criteria for good fixation as given by Wisse et al., ${ }^{19}$ being open sinusoids, intact topography and fine structure for all cell types. Examples of representative images, for both jet and injection perfusion fixation are given in Supplementary Fig. 2A,B (Appendix A).
Thirty-three of the 37 samples showed successful perfusion fixation and the other four samples showed results comparable to immersion fixation.

\section{Sinusoidal endothelial cells}

Perfusion fixation (Fig. 1A) preserved the fine structure of the liver sinusoids (Fig. 1B) and sinusoidal cells including the fenestrae (Fig. 1C) and was successful in 33 of 37 samples. Fenestrae were observed in 17 cases, of which in 11 cases the fenestrae size could be measured. Of these 11 cases, six had NASH and five did not. The median fenestrae size did not differ in samples of patients with NASH versus noNASH [121.0 nm (116.0-138.0) versus $120.0 \mathrm{~nm}$ (93.0-128.0), respectively, $p=0.792]$ (Supplementary Table 2, Appendix A).

Defenestration was identified in 16 cases; for a representative image see Fig. 1D. Significantly less liver biopsy samples of patients with NASH $(9 \%)$ showed defenestration as compared to the noNASH group $(68 \%) \quad(p=0.002)$ (Table 2; Supplementary Table 3, Appendix A). Defenestration was not associated with the severity of steatosis or fibrosis (data not shown, $p>0.3$ for both).

Sinusoidal lipid embolisms, defined as single, large, fat droplets blocking the sinusoid, were observed in nine of the noNASH and one of the NASH samples (45\% vs $8 \%$, respectively, $p=0.050$ ) (Fig. 1E,F).

Table 1 Baseline characteristics of included participants

\begin{tabular}{|c|c|c|c|c|}
\hline & Participants $(n=37)$ & NASH $(n=12)$ & noNASH $(n=25)$ & $p$ value \\
\hline Gender, male/female & $17 / 20(46 \% / 54 \%)$ & $6 / 6(50 / 50 \%)$ & $11 / 14(44 / 56 \%)$ & 0.732 \\
\hline BMI, $\mathrm{kg} / \mathrm{m}^{2}$ & $34.6(6.0)$ & $31.9(5.9)$ & $35.9(5.7)$ & 0.063 \\
\hline Bilirubin, $\mu \mathrm{mol} / \mathrm{L}$ & $11.0(6.7)$ & $14.0(7.8)$ & $9.6(5.8)$ & 0.106 \\
\hline $\mathrm{AP}, \mathrm{U} / \mathrm{L}$ & $119.9(81.9)$ & $170.7(114.8)$ & $95.6(46.1)$ & 0.048 \\
\hline GGT, U/L & $121.4(180.6)$ & $228.7(268.6)$ & $69.8(85.9)$ & 0.068 \\
\hline $\mathrm{ALAT}, \mathrm{U} / \mathrm{L}$ & $69.9(63.9)$ & $118.3(68.9)$ & $46.6(47.1)$ & 0.001 \\
\hline Fasting glucose, ${ }^{\mathrm{a}} \mathrm{mmol} / \mathrm{L}$ & $6.4(3.5)$ & $8.6(6.1)$ & $5.2(0.0)$ & 0.171 \\
\hline Ferritin, ${ }^{\mathrm{a}} \mu \mathrm{g} / \mathrm{L}$ & $238.7(226.1)$ & $335.8(158.1)$ & $202.3(239.6)$ & 0.133 \\
\hline $\mathrm{HDL},{ }^{\mathrm{a}} \mathrm{mmol} / \mathrm{L}$ & $1.18(0.32)$ & $1.3(0.4)$ & $1.1(0.3)$ & 0.258 \\
\hline $\mathrm{LDL},{ }^{\mathrm{a}} \mathrm{mmol} / \mathrm{L}$ & $2.97(1.37)$ & $3.4(1.9)$ & $2.7(1.0)$ & 0.272 \\
\hline Total cholesterol, ${ }^{\mathrm{a}} \mathrm{mmol} / \mathrm{L}$ & $5.03(1.59)$ & $5.7(2.0)$ & $4.7(1.3)$ & 0.075 \\
\hline Triglycerides, ${ }^{\mathrm{a}} \mathrm{mmol} / \mathrm{L}$ & $2.03(1.41)$ & $2.9(2.0)$ & $1.6(0.8)$ & 0.051 \\
\hline \multicolumn{5}{|l|}{ SAF-Steatosis } \\
\hline S0 & $7(19 \%)$ & $0(0 \%)$ & $7(28 \%)$ & \\
\hline $\mathrm{S} 2$ & $6(16 \%)$ & $3(25 \%)$ & $3(12 \%)$ & \\
\hline S3 & $3(8 \%)$ & $3(25 \%)$ & $0(0 \%)$ & \\
\hline \multicolumn{5}{|l|}{ SAF-Activity } \\
\hline A0 & $5(13 \%)$ & $0(0 \%)$ & $5(20 \%)$ & \\
\hline A1 & $18(49 \%)$ & $0(0 \%)$ & $18(72 \%)$ & \\
\hline A2 & $5(13 \%)$ & $3(25 \%)$ & $2(8 \%)$ & \\
\hline A3 & $8(22 \%)$ & $8(67 \%)$ & $0(0 \%)$ & \\
\hline A4 & $1(3 \%)$ & $1(8 \%)$ & $0(0 \%)$ & \\
\hline \multicolumn{5}{|l|}{ SAF-Fibrosis } \\
\hline F0 & $7(19 \%)$ & $0(0 \%)$ & $7(28 \%)$ & \\
\hline $\mathrm{F} 1$ & $16(43 \%)$ & $2(17 \%)$ & $14(56 \%)$ & \\
\hline $\mathrm{F} 2$ & $13(35 \%)$ & $9(75 \%)$ & $4(16 \%)$ & \\
\hline F3 & $1(3 \%)$ & $1(8 \%)$ & $0(0 \%)$ & \\
\hline $\mathrm{F} 4$ & $0(0 \%)$ & $0(0 \%)$ & $0(0 \%)$ & \\
\hline
\end{tabular}

Table 1 shows an overview of the baseline characteristics of the included participants, showing demographic characteristics, blood tests and histology results based on bright-field microscopy.

Continuous variables are expressed as mean \pm standard deviation (SD), and tested using the independent sample T-test. Categorical variables are expressed as absolute figures and percentages and tested using the Chi-square test with Fisher exact when necessary. A two-sided $p$ value of $<0.05$ was considered statistically significant (bold).

NASH, non-alcoholic steatohepatitis.

${ }^{a}$ For these parameters $1-3$ subjects are missing per subgroup NASH or noNASH. 
Table 2 Ultrastructural findings in perfusion fixed human non-alcoholic fatty liver disease samples studied using transmission electron microscopy

\begin{tabular}{|c|c|c|c|c|}
\hline & $\begin{array}{l}\text { Participants } \\
\text { Yes/Total }(\%)\end{array}$ & $\begin{array}{c}\text { NASH } \\
\text { Yes/Total }(\%)\end{array}$ & $\begin{array}{c}\text { noNASH } \\
\text { Yes/Total }(\%)\end{array}$ & $p$ value \\
\hline Steatosis present & $32 / 37(87 \%)$ & $12 / 12(100 \%)$ & $20 / 25(80 \%)$ & \\
\hline Sinusoidal embolism & $10 / 32(31 \%)$ & $1 / 12(8 \%)$ & $9 / 20(45 \%)$ & 0.050 \\
\hline Kupffer cells & $32 / 37(87 \%)$ & $10 / 12(83 \%)$ & $22 / 25(87 \%)$ & \\
\hline Kupffer cell lipofuscin & $16 / 32(50 \%)$ & $5 / 10(50 \%)$ & $11 / 22(50 \%)$ & $>0.999$ \\
\hline Parenchymal lysosomes & $35 / 37(95 \%)$ & $10 / 12(83 \%)$ & $25 / 25(100 \%)$ & \\
\hline Parenchymal lipofuscin & $31 / 35(89 \%)$ & $8 / 10(80 \%)$ & $23 / 25(92 \%)$ & 0.561 \\
\hline Mitochondria & $35 / 37(95 \%)$ & $11 / 12(92 \%)$ & $24 / 25(96 \%)$ & \\
\hline Megamitochondria & $18 / 35(51 \%)$ & $4 / 11(36 \%)$ & $14 / 24(58 \%)$ & 0.289 \\
\hline SCSN & $9 / 37(24 \%)$ & $5 / 12(42 \%)$ & $4 / 25(16 \%)$ & 0.116 \\
\hline
\end{tabular}

NASH, non-alcoholic steatohepatitis; SCSN, single cell steatonecrosis.

${ }^{a}$ Four samples are missing due to insufficient perfusion fixation.

\section{Kupffer cells and stellate cells}

Kupffer cells were observed in 32 cases (Fig. 2A). In these cells, lipofuscin was seen in five of ten NASH (50\%) and 11 of 22 noNASH samples (50\%) ( $p>0.999)$ (Fig. 2B). No apparent signs of storage disease of any substance were found.

In 32 cases, stellate cells were visualised using TEM (Fig. 2C), which showed a normal appearance. Intracellular lipid droplets were observed in 30 of these cases.

Using TEM, fibrosis was found in 24 cases $(75 \%$ vs $60 \%$, NASH vs noNASH, respectively, $p=0.476$ ), of which the majority was seen perisinusoidal. In the bright-field microscopic analysis, 16 cases showed F1, 13 F2, one F3, and seven showed no fibrosis.

\section{TEM analysis of parenchymal cells}

TEM analysis of parenchymal cells could be performed in all 37 perfusion fixed samples. Perfusion fixation showed intact fat droplets within the parenchymal cells (Fig. 3A).

Giant mitochondria in the parenchymal cells, as defined by a large size, abnormal shape and crystalline inclusions, were seen in 18 cases (36\% in NASH vs $58 \%$ in noNASH, $p=0.289$ ) (Fig. 3B). Parenchymal lipofuscin was observed in 31 cases ( $83 \%$ in NASH and $91 \%$ in noNASH, p=0.561) (Fig. 3C, Table 2)

In addition to 'known' ultrastructural findings, a new observation was seen which occurred in a minority of samples (24\%). First, gigantic fat droplets were observed in the middle of the parenchymal cell showing disintegration of the cytoplasm and disruption of the usual arrangement of organelles $(42 \%$ in NASH vs $16 \%$ in noNASH, $p=0.116$ ) (Fig. 4A). In addition, a more complex, but probably further developed situation was observed, in which inflammatory cells, mainly polymorphonuclear granulocytes, and red blood cells, formed a circle around the large fat droplet (Fig. 4B). Cells with these features did not colocalise, but were spread out in the steatotic region. In none of the samples apoptosis was observed. Second, a substance was found in several fat droplets across our study samples; namely a filamentous precipitate, that resembles the filamentous structure of Mallory-Denk bodies (Supplementary Fig. 3, Appendix A).

\section{DISCUSSION}

The current study used perfusion type fixation to explore the ultrastructural changes in liver biopsy samples of NAFLD patients, comparing patients with and without NASH. Successful perfusion was obtained in $89.2 \%$ (33/37) as judged by the following criteria: open sinusoid, intact topography and fine structure for all cell types, including endothelial fenestrae. ${ }^{19,22}$ Significantly less defenestration was found in NASH compared to noNASH samples, while fenestrae size and the presence of other features, i.e., giant mitochondria, did not differ between groups. Furthermore, new structures were identified, i.e., single cell steatonecrosis and inflammatory fat follicles, in a subset of both groups.

This study used perfusion fixation as it allows for proper fixation of all cell types, including the sinusoids and sinusoidal cells, whereas in immersion fixation only the well-fixed peripheral parenchymal cells can be studied. ${ }^{19}$ TEM liver biopsy samples in this study consisted of needle and wedge liver biopsies; therefore, two different perfusion techniques were used. Injection fixation was used in wedge biopsies. With this technique the fixative is injected into the wedge biopsy. ${ }^{19}$ It is not possible to use this technique in needle biopsies, due to the smaller tissue sample; therefore, the technique of jet fixation, spraying the fixative onto the biopsy sample, was used in the needle biopsies. ${ }^{20}$ Vice versa, it is not possible to use jet fixation in wedge biopsies as the size of the wedge biopsies is too large and perfusion would not take place. The results of both jet and injection fixation are comparable to perfusion through the portal vein in animal studies. ${ }^{23}$

In the current study, special attention was paid to the LSECs and fenestrae since they serve as a sieve and play an important role in the transport of lipids across the sinusoid. The hypothesis of the study was that fenestrae size and number decrease as the severity of NAFLD increases. Unexpectedly, we showed that defenestration was more prevalent in the noNASH samples compared to NASH samples. In addition, defenestration was not related to the degree of steatosis or fibrosis.

Based on the current findings and the fact that defenestration is seen more in simple steatosis, instead of in NASH, one could argue that defenestration is a protective mechanism to handle the excess in lipids and perhaps other toxic substances, such as bacterial products. If defenestration plays a role in inhibiting the entry of chylomicron remnants into the space of Disse, ${ }^{18}$ it could protect the liver from receiving more lipids. On the other hand, defenestration also impairs the passage of VLDL excretion towards the sinusoidal lumen, thereby causing hepatic lipid accumulation. ${ }^{14}$ Furthermore, inhibition of lipid uptake by defenestration has been proposed to lead to stimulation of $d e$ 

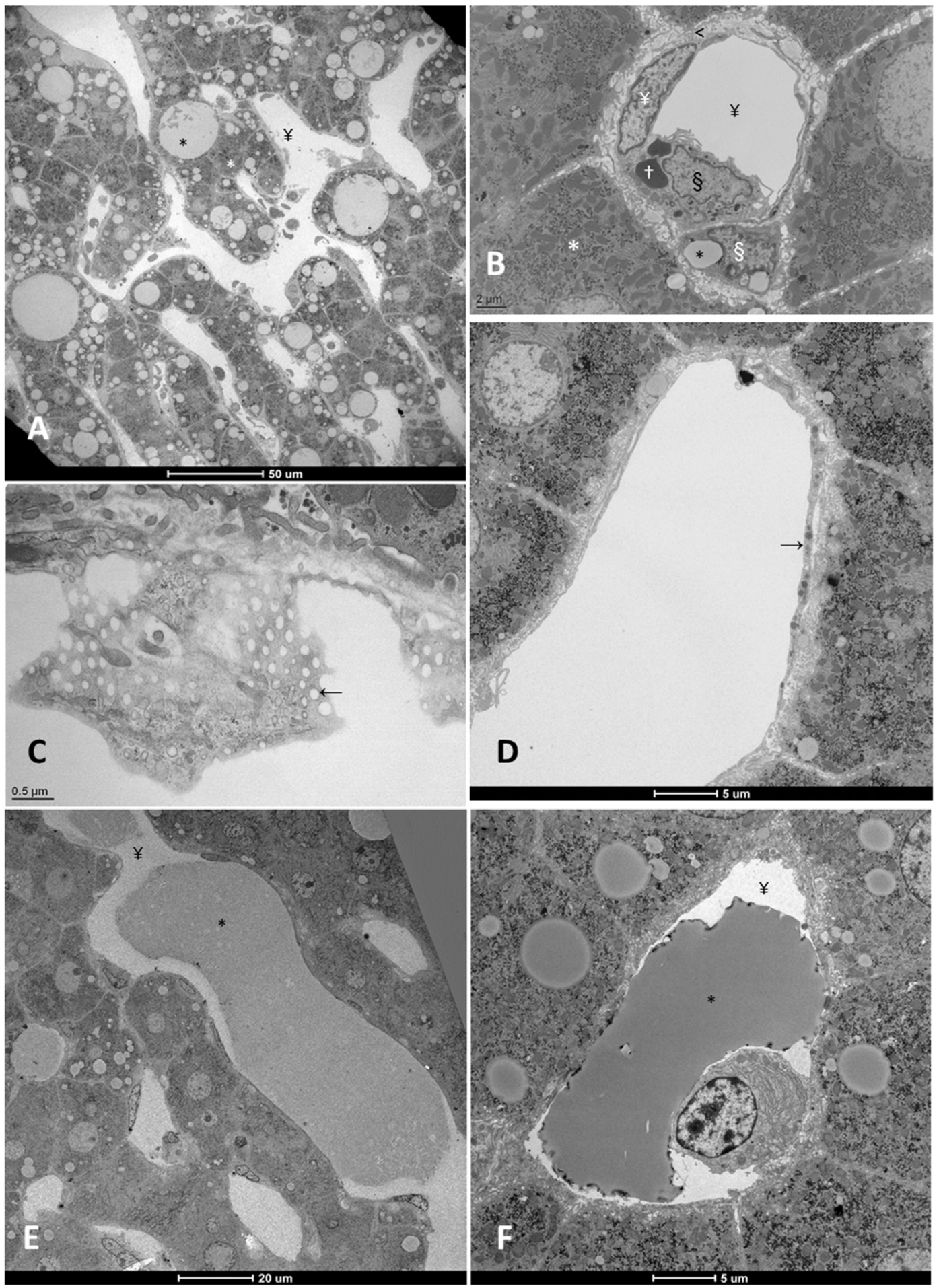

Fig. 1 (A) Low magnification transmission electron microscopy (TEM) of a non-alcoholic steatohepatitis (NASH) liver needle biopsy sample, fixed by jet fixation, showing parenchymal cells (white $*$ ) with different sizes of fat droplets $(*)$ and open sinusoids (¥). Note the openness of the sinusoids and the preserved topography and fine structure of all cells present. (B) Four cell types are united in this figure of a jet-fixed noNASH liver needle biopsy, showing parenchymal cells (white *) together with one liver sinusoidal endothelial cell (white $¥$ ), a Kupffer cell (§) and a stellate cell (white $\S$ ) (respectively, from top to bottom), together surrounding an open sinusoidal lumen (¥) free of plasma and blood cells. This shows the normal topographical arrangement of these cell types that is lost in immersion fixation. A normal space of Disse $(<)$ separates the endothelial lining from the parenchymal cells. The Kupffer cell shows endocytosed electron dense material (white $\dagger$ ) and the stellate cell (previously named fat-storing cell or Ito-cell) contains two fat droplets $(*)$. (C) Higher magnification from a jet fixed needle biopsy of a NASH patient. Fenestrae within the endothelial lining are observed when the lining is obliquely cut during sectioning for TEM. Because of the isotonicity of the fixative and the inclusion of the cells in epoxy resin, the size of the fenestrae is presumed to be preserved to represent the size in vivo. Fenestrae are grouped in thin, slightly electron dense cytoplasmic sieve plates (black arrow). (D) TEM figure of a defenestrated sinusoid of a noNASH patient (injection fixation). There are no or hardly any fenestrae in well-fixed livers with preserved fine structured details (black arrow). This condition is called pseudocapillarisation, capillarisation or defenestration by some authors (needle biopsy, jet fixation). (E,F) Fat droplets $(*)$ in the liver sinusoids ( $¥)$ of noNASH patients (sinusoidal lipid embolism) (injection fixation). Phagocytoses of whole fat droplets or parts of it by, e.g., Kupffer cells or other cells were not observed. Furthermore, it is unclear how long fat droplets remain in the sinusoids before being degraded. 

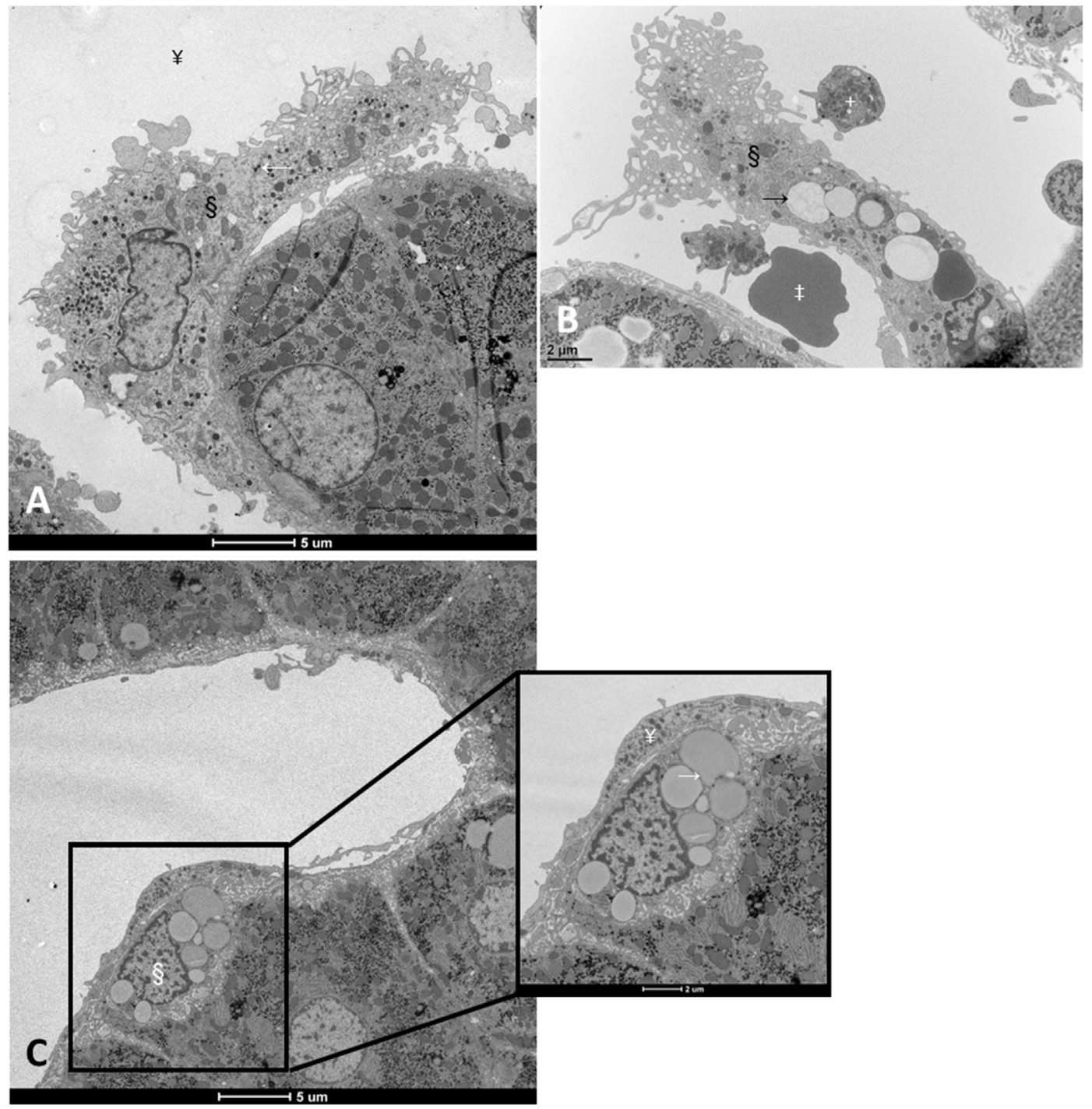

Fig. 2 (A) Transmission electron microscopy (TEM) of a Kupffer cell ( $\$$ ) on top of a parenchymal cell. Kupffer cells have an irregular shape and a rough surface. These cells are exposed to the sinusoidal (¥) blood flow and phagocytose foreign cells and materials present in the blood. This particular Kupffer cell shows the absence of phagocytosed material, but displays the presence of numerous small lysosomes which are the electron dense granules in the cytoplasm (white arrow) (injection fixation of wedge biopsy, noNASH patient). (B) TEM of a Kupffer cell (§) in a jet fixed needle biopsy of a noNASH patient, showing the presence of phagocytosed material of different density (lipofuscin) (black arrow). Other cell types, such as a red blood cell (white $\$$ ) and several white blood cells (white + ), probably lymphocytes, are present. (C) TEM (needle biopsy, noNASH patient, jet fixation) of a stellate cell (white $\S$ ) which is an inhabitant of the space of Disse. These cells are always covered by the endothelial lining (white $¥$ in inset); therefore, the cell is shielded from the sinusoidal blood flow. Within the cytoplasm of the cell we observe fat droplets (white arrow, inset) that can vary in number and size. Inset: enlargement of the stellate cell.

novo lipid (DNL) synthesis and thereby induces steatosis. ${ }^{18}$ This is due to the fact that hydroxymethylglutaryl-CoA reductase (HMGCR), the rate limiting enzyme for hepatocyte cholesterol synthesis, is no longer inhibited when chylomicron remnants and dietary cholesterol do not cross the fenestrae, thereby activating endogenous cholesterol synthesis in hepatocytes. ${ }^{24}$ However, one could argue that in case of defenestration, DNL could be performed at a pace that does not overwhelm beta oxidation and re-esterification within the liver. This would enable the liver to store the fat as triglycerides in lipid droplets, instead of metabolising them. This fits the current view on the pathophysiology of NAFLD, which proposes that NASH and simple steatosis are independent twin conditions. Steatosis in NASH is being seen as a benign epiphenomenon rather than a causal factor of inflammation and fibrosis, which are the two main hallmarks of NASH. ${ }^{25}$ In NASH, the metabolisation of free fatty acids and cholesterol leads to formation of toxic lipid species, however, the underlying mechanisms of the formation of lipotoxic intermediates at molecular levels remain unknown $^{26}$; whereas triglyceride accumulation in the form of lipid droplets in simple steatosis may serve as a nontoxic, safer form of storage for lipids in the liver.

When comparing fenestrae size between NASH and noNASH samples, we found no significant difference [121.0 $\mathrm{nm}(116.0-138.0)$ in NASH and 120.0 (93.0-128.0) $\mathrm{nm}$ in noNASH]. Fenestrae size in humans without liver pathology show a diameter of $107( \pm 1.5) \mathrm{nm}^{28}$

Our findings in human samples are not in line with the animal model findings of Herrnberger et al., ${ }^{18}$ studying PLVAP deficient mice. PLVAP is known to be critical for the development of fenestrae. PLVAP deficient mice developed extensive multivesicular steatosis, but also steatohepatitis and fibrosis at around 3 weeks of life. ${ }^{18}$ This stresses the added value of human studies as animal models do not recapitulate 

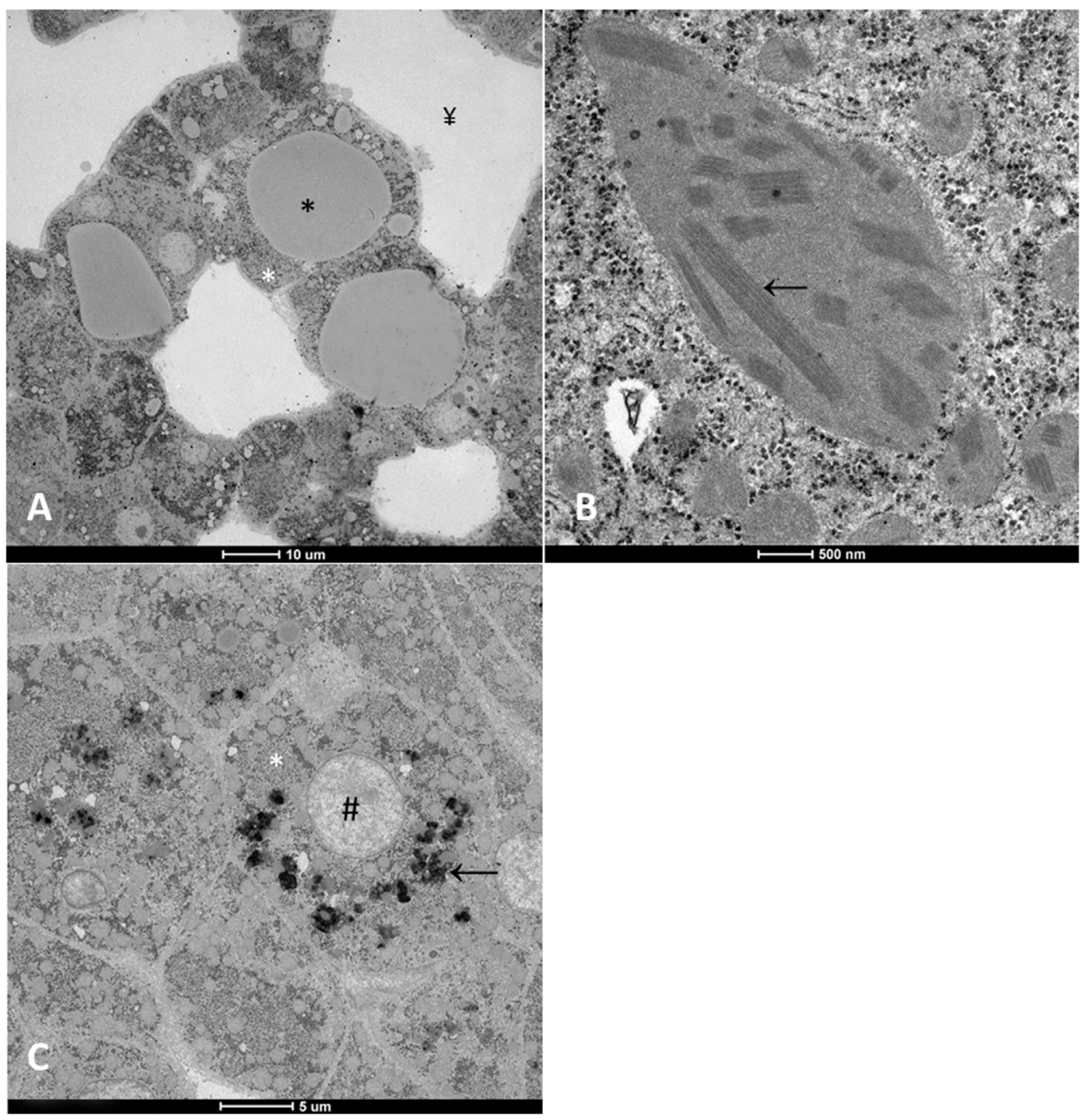

Fig. 3 (A) In steatosis, the fat droplets (*) in parenchymal cells (white *) can grow to proportions that largely exceed the size of a normal parenchymal cell. In most cases we only find a single large fat droplet per cell. Open sinusoids (¥) are present at several sides of the parenchymal cells. (wedge biopsy, injection fixation, noNASH patient). (B) A giant mitochondrion is shown (wedge biopsy, injection fixation, noNASH patient). The size of giant mitochondria varies between normal and gigantic. Always, crystals (black arrow) can be recognised in the matrix of these intriguing organelles. Apart from the variety in size, the giants also show different shapes and content. They can be round, oval, cylindric, or branched. Their occurrence is regional, but it is not yet clear whether their presence is zonal (portal or central). They never populate all parenchymal cells. They are absent in the livers of experimental animals like rats, mice, rabbits, guinea pigs. (C) Transmission electron microscopy of a parenchymal cell in which the nucleus (\#) is surrounded by lipofuscin containing residual bodies (black arrow), which are in fact old (retired) lysosomes with reduced or even absent digesting capacity. Normally lysosomes are oriented to the bile canaliculi, but in the case of larger numbers, they are more dispersed in the cell (wedge biopsy, injection fixation, noNASH patient).

all pathological features of human NAFLD or NASH. Furthermore, they do not capture the large inter-individual variation with regard to host genetics and environmental factors. $^{29}$

The presence of giant mitochondria in this study showed no difference between patients with and without NASH. These findings are in line with Ahishali et al. ${ }^{13}$ Giant mitochondria have been considered to represent an intermediate phase in evolution of steatohepatitis, regardless of the source of hepatic fat deposition. ${ }^{12}$ However, their exact role in the pathophysiology in NAFLD is unclear. Based on our findings, they might play a role in NAFLD, but that role is not limited to steatohepatitis.

In the current study, new structures were identified and described, namely single cell steatonecrosis (SCSN) and inflammatory fat follicles (IFF). Single cell steatonecrosis
(SCSN) shows an enormous fat droplet in a single parenchymal cell in a well advanced stage of necrosis. In addition, this condition may further develop into a situation where the large central single fat droplet is surrounded with a monolayer of inflammatory cells, such as polymorphonuclear granulocytes, but also by red blood cells. We propose the term 'inflammatory fat follicle' (IFF) for this feature. It has been shown that accumulating lipid droplets can mechanically distort the intracellular environment, as lipid droplets are stiffer than the surrounding cytosol, thereby leading to cellular damage. Baldini et al. ${ }^{30}$ have studied the biomechanics of cultured steatotic hepatocytes and showed that hepatocyte cell stiffness and cell viability are directly influenced by the extent of triglyceride accumulation and the size of lipid droplets. Furthermore, the swelling of parenchymal cells may lead to the narrowing of local sinusoids, and contribute to vascular resistance. The size of the fat droplets 

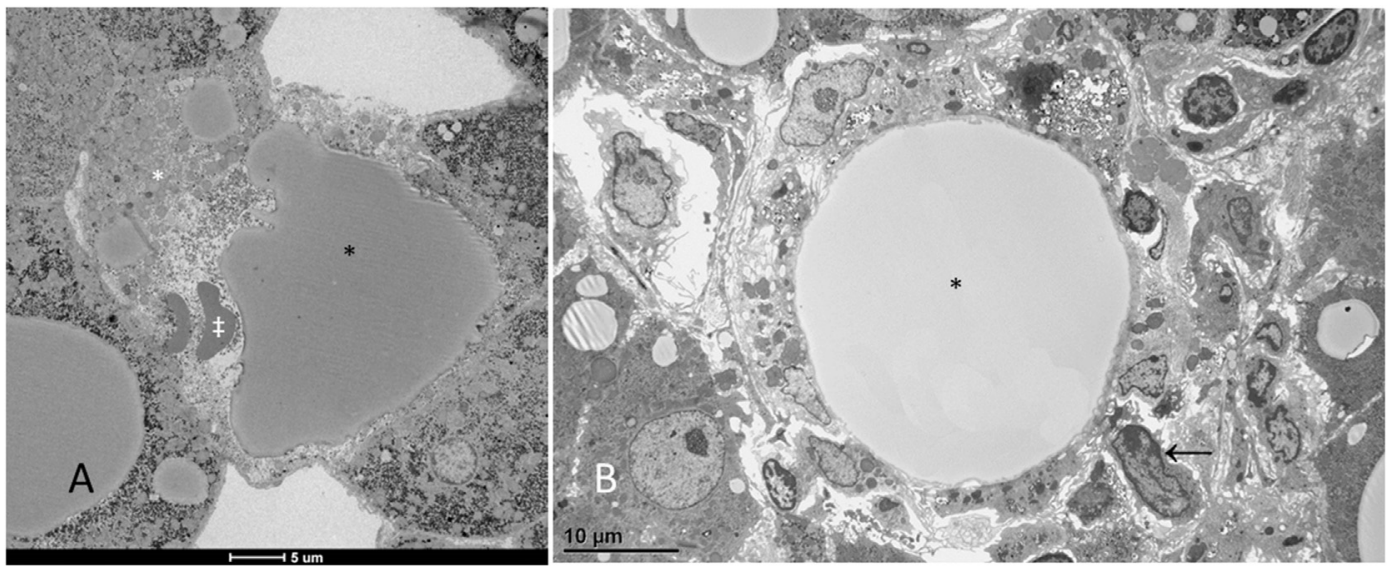

Fig. 4 (A) Transmission electron microscopy (TEM) with a clear example of single cell steatonecrosis (SCSN). The cell shows disorganisation of the cytoplasm resulting in a free suspension of organelles (white *), probably in Brownian movement. A large fat droplet $(*)$ is supposed to have introduced necrosis in this parenchymal cell. The organelles are more or less confined within the boundaries of the cell or the still intact endothelial lining. Two red blood cells (white $\ddagger$ ) are present. It is not yet clear to what extent this situation contributes to developing the inflammatory aspect of steatohepatitis (wedge biopsy, injection fixation, noNASH patient). (B) TEM of an inflammatory fat follicle (IFF). Remarkably, in IFF a monolayer of polymorphonuclear neutrophils (PMNs) (black arrow) is surrounding the fat droplet $(*)$. The presence of PMNs suggest that these cells take care of digesting the large fat droplet and at the same time might contribute to the inflammatory process (needle biopsy, jet fixation, NASH patient).

in severe steatosis exceeds the diameter of parenchymal cells and sinusoids considerably. ${ }^{31}$ Previous research in rats showed an increase in intrahepatic resistance with haemodynamic impact in relation to severe steatosis. ${ }^{32}$ This study observed sinusoidal lipid embolisms in both NASH and noNASH patients. One could argue that these can also be a contributing factor to vascular resistance.

When interpreting the results of this study, it is important to note several limitations. Liver biopsy samples were obtained via two different routes. Needle biopsy samples were collected via percutaneous liver biopsies in outpatients with liver enzyme abnormalities as part of clinical care. Wedge biopsy samples were collected during bariatric surgery in overweight patients. Consequently, the liver biopsies originate from different parts of the liver, more peripherally for the wedge biopsies versus more centrally for the needle biopsies. Furthermore, it should be noted that the patients who underwent bariatric surgery followed a calorie restricted diet before surgery. ${ }^{33}$ Defenestration has been linked to diets varying in content of macronutrients in animal models, ${ }^{15,34}$ suggesting that a diet based on caloric restriction reduces defenestration. However, a larger part of the noNASH group underwent bariatric surgery and a calorie restricted diet, still showing more defenestration compared to the NASH group. Therefore, we think that this calorie restricted diet did not have a major impact on our results.

It should also be noted that only small parts of the liver are evaluated by electron microscopy. It is known that histological lesions in NAFLD are unevenly distributed throughout the liver and a sampling error can occur when studying lesions using bright-field microscopy. ${ }^{35}$ Therefore, it is difficult to quantify parameters for the total organ. In seven patients with steatosis on imaging, steatosis could not be confirmed, when revising the liver biopsy with bright-field microscopy for the current study, further supporting that sampling errors cannot be excluded. However, when excluding these samples from the analysis for defenestration, the difference between groups remained significant ( $p=0.005$, data not shown).
To our knowledge, this is the first study on fenestrae in perfusion fixed human NAFLD liver samples using TEM. Previous studies used immersion fixation, ${ }^{8-13,36,37}$ leaving the centre of the biopsy improperly fixed, which does not allow for accurate analysis of sinusoids and sinusoidal cells. ${ }^{20}$ Second, a relatively large sample size was included, directly comparing NASH vs noNASH samples, whereas other studies included smaller sample sizes, ${ }^{8-12,37}$ or studied animal models. ${ }^{18,38}$

\section{CONCLUSION}

We demonstrated that perfusion type-fixation techniques are feasible, for both needle and wedge liver biopsies, in human NAFLD liver samples. By using these techniques, new ultrastructural details, such as SCSN and IFF, were revealed. An almost complete lack of fenestrae (defenestration) in NAFLD patients without NASH was found. Defenestration may be a protective mechanism to handle the excess in lipids and perhaps other toxic substances and may serve as a possible explanation why some patients develop NASH and others do not. Future studies are needed to further investigate the role of these findings within the multifactorial aetiology of NASH.

Conflicts of interest and sources of funding: The authors state that there are no conflicts of interest to disclose.

\section{APPENDIX A. SUPPLEMENTARY DATA}

Supplementary data to this article can be found online at https://doi.org/10.1016/j.pathol.2020.07.018.

Address for correspondence: Dr P. L. M. Verhaegh, Maastricht University Medical Center, Department of Internal Medicine, Division of Gastroenterology and Hepatology, P. Debyelaan 25, PO Box 5800, 6202 AZ, Maastricht, the Netherlands. E-mail: Pauline.verhaegh@mumc.nl

\section{References}

1. Younossi ZM, Koenig AB, Abdelatif D, Fazel Y, Henry L, Wymer M. Global epidemiology of nonalcoholic fatty liver disease-Meta-analytic 
assessment of prevalence, incidence, and outcomes. Hepatology 2016; 64: 73-84.

2. Bellentani S, Scaglioni F, Marino M, Bedogni G. Epidemiology of nonalcoholic fatty liver disease. Dig Dis 2010; 28: 155-61.

3. Chalasani N, Younossi Z, Lavine JE, et al. The diagnosis and management of non-alcoholic fatty liver disease: practice guideline by the American Gastroenterological Association, American Association for the Study of Liver Diseases, and American College of Gastroenterology. Gastroenterology 2012; 142: 1592-609.

4. Hashimoto E, Taniai M, Tokushige $\mathrm{K}$. Characteristics and diagnosis of NAFLD/NASH. J Gastroenterol Hepatol 2013; 28 (Suppl 4): 64-70.

5. McCullough AJ. Pathophysiology of nonalcoholic steatohepatitis. J Clin Gastroenterol 2006; 40 (Suppl 1): S17-29.

6. Ong JP, Pitts A, Younossi ZM. Increased overall mortality and liverrelated mortality in non-alcoholic fatty liver disease. J Hepatol 2008; 49: $608-12$.

7. Friedman SL, Neuschwander-Tetri BA, Rinella M, Sanyal AJ. Mechanisms of NAFLD development and therapeutic strategies. Nat Med 2018; 24: 908-22.

8. Fukuo Y, Yamashina S, Sonoue H, et al. Abnormality of autophagic function and cathepsin expression in the liver from patients with nonalcoholic fatty liver disease. Hepatol Res 2014; 44: 1026-36.

9. Iancu TC, Manov I, Shaoul R, Haimi M, Lerner A. What's in a name? "Lipolysosome": ultrastructural features of a lipid-containing organelle. Ultrastruct Pathol 2013; 37: 293-303.

10. Caldwell S, Ikura Y, Dias D, et al. Hepatocellular ballooning in NASH. J Hepatol 2010; 53: 719-23.

11. Caldwell SH, de Freitas LA, Park SH, et al. Intramitochondrial crys talline inclusions in nonalcoholic steatohepatitis. Hepatology 2009; 49 $1888-95$.

12. Caldwell SH, Swerdlow RH, Khan EM, et al. Mitochondrial abnormalities in non-alcoholic steatohepatitis. J Hepatol 1999; 31: 430-4.

13. Ahishali E, Demir K, Ahishali B, et al. Electron microscopic findings in non-alcoholic fatty liver disease: is there a difference between hepatosteatosis and steatohepatitis? J Gastroenterol Hepatol 2010; 25: 619-26.

14. Hammoutene A, Rautou PE. Role of liver sinusoidal endothelial cells in non-alcoholic fatty liver disease. J Hepatol 2019; 70: 1278-91.

15. Cogger VC, Mohamad M, Solon-Biet SM, et al. Dietary macronutrients and the aging liver sinusoidal endothelial cell. Am J Physiol Heart Circ Physiol 2016; 310: H1064-70.

16. Zhang Q, Liu J, Liu J, et al. oxLDL induces injury and defenestration of human liver sinusoidal endothelial cells via LOX1. J Mol Endocrinol 2014: 53: $281-93$.

17. Dobbs BR, Rogers GW, Xing HY, Fraser R. Endotoxin-induced defenestration of the hepatic sinusoidal endothelium: a factor in the pathogenesis of cirrhosis? Liver 1994; 14: 230-3.

18. Herrnberger L, Hennig R, Kremer W, et al. Formation of fenestrae in murine liver sinusoids depends on plasmalemma vesicle-associated protein and is required for lipoprotein passage. PLoS One 2014; 9 : e115005.

19. Wisse E, Braet F, Duimel H, et al. Fixation methods for electron microscopy of human and other liver. World J Gastroenterol 2010; 16: $2851-66$.
20. Vreuls C, Wisse E, Duimel H, et al. Jet-fixation: a novel method to improve microscopy of human liver needle biopsies. Hepatology 2014; 59: 737-9.

21. Bedossa P, Poitou C, Veyrie N, et al. Histopathological algorithm and scoring system for evaluation of liver lesions in morbidly obese patients. Hepatology 2012; 56: 1751-9.

22. Wisse E, Braet F, Luo D, et al. Structure and function of sinusoidal lining cells in the liver. Toxicol Pathol 1996; 24: 100-11.

23. Wisse E. An electron microscopic study of the fenestrated endothelial lining of rat liver sinusoids. J Ultrastruct Res 1970; 31: 125-50.

24. Fraser R, Cogger VC, Dobbs B, et al. The liver sieve and atherosclerosis. Pathology 2012; 44: 181-6.

25. Yilmaz Y. Review article: is non-alcoholic fatty liver disease a spectrum, or are steatosis and non-alcoholic steatohepatitis distinct conditions? Aliment Pharmacol Ther 2012; 36: 815-23.

26. Neuschwander-Tetri BA. Hepatic lipotoxicity and the pathogenesis of nonalcoholic steatohepatitis: the central role of nontriglyceride fatty acic metabolites. Hepatology 2010; 52: 774-88.

27. Bessone F, Razori MV, Roma MG. Molecular pathways of nonalcoholic fatty liver disease development and progression. Cell Mol Life Sci 2019; 76: $99-128$.

28. Wisse E, Jacobs F, Topal B, Frederik P, De Geest B. The size of endothelial fenestrae in human liver sinusoids: implications for hepatocyte-directed gene transfer. Gene Ther 2008; 15: 1193-9.

29. Castro RE, Diehl AM. Mistakes in mouse models of nonalcoholic steatophepatitis and how to avoid them. UEG Educ 2018; 18: 30-4.

30. Baldini F, Bartolozzi A, Ardito M, et al. Biomechanics of cultured hepatic cells during different steatogenic hits. J Mech Behav Biomed Mater 2019; 97: 296-305.

31. Wisse E, De Zanger RB, Charels K, Van Der Smissen P, McCuskey RS. The liver sieve: considerations concerning the structure and function of endothelial fenestrae, the sinusoidal wall and the space of Disse. Hepatology 1985; 5: 683-92.

32. Francque S, Laleman W, Verbeke L, et al. Increased intrahepatic resistance in severe steatosis: endothelial dysfunction, vasoconstrictor overproduction and altered microvascular architecture. Lab Invest 2012 92: $1428-39$.

33. De Munck TJI, Verhaegh PLM, Verbeek J, et al. Crashing NASH in patients listed for bariatric surgery. Obes Surg 2019; 29: 1012-4.

34. Jamieson HA, Hilmer SN, Cogger VC, et al. Caloric restriction reduces age-related pseudocapillarization of the hepatic sinusoid. Exp Geronto 2007; 42: 374-8.

35. Ratziu V, Charlotte F, Heurtier A, et al. Sampling variability of liver biopsy in nonalcoholic fatty liver disease. Gastroenterology 2005; 128: $1898-906$.

36. Le TH, Caldwell SH, Redick JA, et al. The zonal distribution of megamitochondria with crystalline inclusions in nonalcoholic steatohepatitis. Hepatology 2004; 39: 1423-9.

37. Lotowska JM, Sobaniec-Lotowska ME, Bockowska SB, Lebensztejn DM. Pediatric non-alcoholic steatohepatitis: the first report on the ultrastructure of hepatocyte mitochondria. World J Gastroenterol 2014; 20: 4335-40.

38. Kanai M. Ultrastructural and biochemical studies of lipolysis by lipolysosomes in chick hepatocytes. Cell Tissue Res 1989; 255: 559-65. 